\title{
The vacuum bubbles in de Sitter background and black hole pair creation
}

\author{
Bum - Hoon Lee'1 and Wonwoo Lę2 \\ Department of Physics and BK21 Division, and Center for Quantum Spacetime, Sogang University, \\ Seoul 121-742, Korea
}

\begin{abstract}
We study the possible types of the nucleation of vacuum bubbles. We classify vacuum bubbles in de Sitter background and present some numerical solutions. The thin-wall approximation is employed to obtain the nucleation rate and the radius of vacuum bubbles. With careful analysis we confirm that Parke's formula is also applicable to the large true vacuum bubbles. The nucleation of the false vacuum bubble in de Sitter background is also evaluated. The tunneling process in the potential with degenerate vacua is analyzed as the limiting cases of the large true vacuum bubble and false vacuum bubble. Next, we consider the pair creation of black holes in the background of bubble solutions. We obtain static bubble wall solutions of junction equation with black hole pair. The masses of created black holes are uniquely determined by the cosmological constant and surface tension on the wall. Finally, we obtain the rate of pair creation of black holes.

PACS numbers: 98.80.Cq, 04.70.Dy, 11.27.+d
\end{abstract}

\footnotetext{
${ }^{1}$ email:bhl@sogang.ac.kr

${ }^{2}$ email:warrior@sogang.ac.kr
} 


\section{Introduction}

The aims of this paper are to obtain the nucleation rate and the radius of vacuum bubbles and to study the pair creation of black holes in the presence of a bubble wall. The processes of bubble nucleations and black hole productions may mimic Wheeler's spacetime foam structure [1] in the very early universe, which represents the spacetime no longer smooth at the Planck scale. Bubble nucleation [2, 3, 4, 5] and Hawking-Moss type transition [6] may occur in the early universe. They may play an important role in selecting out our universe or inflation [7]. In addition, the creation of black holes may also play an important role, as a topology changing process, in the spacetime foam structure. Some bubbles may have black holes. Some of them may be nucleated in the presence of a black hole, which is studied by Hiscock [8] and Berezin et al. [9], where the black hole act as an effective nucleation center for a bubble formation. Others may cause pair creation of black holes. On the other hand, the string theory landscape paradigm has many stable and metastable vacua [10]. So the tunneling process becomes a remarkable event in this framework as well as eternal inflation [11].

We would like to understand the mechanism how the complicated spacetime structure could be created and tunneling process occur. Can the theory tell us which bubble is our universe if we live in one? Why the particular bubble is favored? These questions are related to the cosmological constant problem and anthropic constraints. True vacuum bubbles can be nucleated within false vacuum as well as false vacuum bubbles can be nucleated within true vacuum. Black hole can also be created in these situation. These events cause the complicated structure of the early universe. Moreover, we may live in one bubble today. Our study show that the important thing is the cosmological constant. We will discuss that later in more detail. We want to classify all possible types of bubbles. It is related to the cosmological constant in our framework.

In this paper, we directly compute the nucleation rate of possible vacuum bubbles using thin-wall approximation. We will classify the true vacuum bubble in three classes : "small" bubble - "large" background, "half" bubble - "half" background, and "large" bubble - "small" background, the terminology will be defined later. In Ref. [12], Parke evaluated the nucleation rate and the radius of a bubble with an arbitrary cosmological constant. In our terminology, the bubble in Ref. [12] corresponds to a "small" true vacuum bubble within a "large" false vacuum background. We carefully examine his formula to the cases of large true vacuum bubbles, degenerate case, and false vacuum bubbles while restricting the background as de Sitter. Another method to account for the initial quantum field state as the conformal vacuum with gravity was proposed [13. The authors studied the Hawking-Moss transition as a limit of constrained instantons and Lee-Weinberg transition [14] as a false vacuum bubble nucleation. The vacuum tunneling with a DBI action is considered in Coleman-de Luccia type [15] and Hawking-Moss type transition [16]. A new method to calculate the tunneling wave function that describes vacuum decay was studied by Gen and Sasaki [17]. The tunneling using junction condition and shell-mediated tunneling was studied [18]. The effect of the Gauss-Bonnet term on vacuum decay was also studied [19]. The mechanism of the nucleation of a false vacuum 
bubble due to nonminimal coupling was obtained [20]. The dynamics of a false vacuum bubble with an effective negative tension due to the coupling was also studied in Re. [21] (for a breathing bubble, see Ref. [22] and dynamics of a vacuum bubble, see Ref. [23]). It was proposed that the observed accelerated expansion of the universe is driven by the false vacuum energy of a colored scalar field in Ref. [24]). Recently, Marvel and Wesley studied thin-wall instanton with negative tension wall and its relation to Witten's bubble of nothing [25]. The negative boundary tension brane was analyzed in Ref. [26]. In Ref. [27], the authors discussed the possible instanton solutions in Euclidean de Sitter space. They studied tunneling solution with the action having only Einstein-Hilbert term and cosmological term without scalar field. Brown and Weinberg studied thermal derivation of the Coleman-De Luccia tunneling [28]. The authors clarified the meaning of the bounce solution itself as well as oscillating bounce solutions [29]. HawkingMoss transition was analyzed as a thermal fluctuation [30]. Recently, the relation between Coleman-de Luccia and Hawking-Moss transition was studied in Ref. [31].

Next, we will consider black hole pair creation. The vacuum of a strong field can decay due to the Schwinger mechanism 32 for particle production. Particle production is one of the decaying methods of the background field or given vacuum energy (for recent works, see Ref. [33]). For the black hole creation, Gross, Perry, and Yaffe [34 obtained the nucleation rate for black holes in a thermal bath. The nucleation of black holes in the de Sitter universe with a semiclassical instability was considered by Ginsparg and Perry [35]. The pair creation of black holes was originally obtained by Gibbons [36]. Wu studied a single black hole creation at the birth of the universe using the no-boundary proposal [37] (for other frameworks, see Ref. [38]). In Ref. [39, the authors exhibited the new decaying process of the cosmological constant by thermal production of membranes. Eventually, the membrane collapses into a black hole. Black hole pair creation in the early universe was extensively investigated [40, 41] and in other frameworks [42, 43, 44, 45]. Caldwell, Chamblin, and Gibbons studied black hole pair creation in the presence of a domain wall [46] using the cut-and-paste procedure, where the background has vanishing cosmological constant and the probability was obtained. The repulsive property of the domain wall give rise to black hole pair creation. The calculating method in their work will be used in our paper. However, our solutions give rise to the background for the black hole pair creation with a bubble wall more naturally.

The outline of this paper is as follows: In Sec. 2 we investigate the possible types of the nucleation of vacuum bubbles. We classify true vacuum bubbles in de Sitter background into nine types depending on whether the bubble and the background size are small, half, or large. We also present some numerical solutions corresponding to large bubbles with small background. We compute analytically the nucleation rate and the radius of false vacuum bubbles using the thin-wall approximation. We extend the analysis to the large true vacuum bubbles and show that the formula in Ref. [12] still holds in this case. Our approach is different from those in Refs. [27, 47] without scalar field. We also consider more variety of types. The relation to the Hawking-Moss transition will be mentioned. Furthermore, we explore the case of degenerate vacua in de Sitter space. The numerical solution of this tunneling was obtained in Ref. [29]. This case corresponds to a special transitions of the nucleation of a true vacuum bubble and 
a false vacuum bubble. We compare this transition with the formation of a domain wall. The transition rate and the radius of the bubble are presented. The deformed bulk shape causes the contribution of bulk part in the action. In Sec. 3 we present the solution of pair creation of black holes in the presence of a bubble wall. Some possible types in previous section are adopted as the background space for pair creation of black holes. We obtain static bubble wall solutions of junction equation with black hole pair. The mass of created black holes is uniquely determined by given cosmological constant and surface tension on the wall. Finally, we obtain the rate of pair creation of black holes. We summarize and discuss our results in Sec. 4 .

\section{The nucleation rate and the radius of vacuum bubbles in de Sitter space}

Let us consider the action

$$
S=\int_{\mathcal{M}} \sqrt{-g} d^{4} x\left[\frac{R}{2 \kappa}-\frac{1}{2} \nabla^{\alpha} \Phi \nabla_{\alpha} \Phi-U(\Phi)\right]+\oint_{\partial \mathcal{M}} \sqrt{-h} d^{3} x \frac{K}{\kappa},
$$

where $\kappa \equiv 8 \pi G, g \equiv \operatorname{det}_{\mu \nu}$, and the second term on the right-hand side is the boundary term [48. $U(\Phi)$ is the scalar field potential with two non-degenerate minima with lower minima at $\Phi_{T}$ and higher minima at $\Phi_{F}, R$ denotes the Ricci curvature of spacetime $\mathcal{M}$, and $K$ is the trace of the extrinsic curvature of the boundary $\partial \mathcal{M}$. Here we adopt the notations and sign conventions of Misner, Thorne, and Wheeler [49].

The bubble nucleation rate or the decay rate of background vacuum is semiclassically given by $\Gamma / V=A e^{-B / \hbar}$, where $B$ is the difference between Euclidean action corresponding to bubble solution and that of the background and the prefactor $A$ is discussed in Ref. [50], that with some gravitational corrections in Ref. [51]. We are interested in finding the coefficient $B$.

We will take $O(4)$ symmetry for both $\Phi$ and the spacetime metric $g_{\mu \nu}$, expecting its dominant contribution [52]. The general $O(4)$-symmetric Euclidean metric is written by

$$
d s^{2}=d \eta^{2}+\rho^{2}(\eta)\left[d \chi^{2}+\sin ^{2} \chi\left(d \theta^{2}+\sin ^{2} \theta d \phi^{2}\right)\right] .
$$

The Euclidean field equations for $\Phi$ and $\rho$ have the form

$$
\begin{gathered}
\Phi^{\prime \prime}+\frac{3 \rho^{\prime}}{\rho} \Phi^{\prime}=\frac{d U}{d \Phi}, \\
\rho^{\prime \prime}=-\frac{\kappa}{3} \rho\left(\Phi^{\prime 2}+U\right),
\end{gathered}
$$

respectively and the Hamiltonian constraint is given by

$$
\rho^{\prime 2}-1-\frac{\kappa \rho^{2}}{3}\left(\frac{1}{2} \Phi^{\prime 2}-U\right)=0,
$$


where the prime denotes the differentiation with respect to $\eta$. We will consider only the case with initial de Sitter space. The boundary conditions for the bounce are

$$
\left.\frac{d \Phi}{d \eta}\right|_{\eta=0}=0,\left.\quad \frac{d \Phi}{d \eta}\right|_{\eta=\eta_{\max }}=0, \quad \rho_{\eta=0}=0, \text { and } \rho_{\eta=\eta_{\max }}=0,
$$

where $\eta_{\max }$ is a finite value in Euclidean de Sitter space. The asymptotic value of $\Phi(\eta)$ is given by $\lim _{\eta \rightarrow \eta_{\max }} \Phi(\eta) \simeq \Phi_{F / T}$, where $\Phi_{F}$ is for a true vacuum bubble nucleation and $\Phi_{T}$ is for a false one. Because of the finiteness of $\eta_{\max }, \Phi\left(\eta_{\max }\right)$ is exponentially approaching to but not reaching $\Phi_{F / T}$.

Now, we assume the thin-wall approximation scheme to evaluate $B$. The validity of that in the case of a true vacuum bubble has been examined [53]. The Euclidean action can be divided into three parts: $B=B_{\text {in }}+B_{\text {wall }}+B_{\text {out }}$. The configuration of the outside of the wall will not be changed before and after bubble formation. Thus, $B_{\text {out }}=0$. So we only need to consider the contribution of the wall and inside part. On the wall [4], $B_{\text {wall }}=2 \pi^{2} \bar{\rho}^{3} S_{o}$, where $S_{o}$ is the tension of the wall. The action has a stationary point, which determines the radius $\bar{\rho}$ of the bubble. The contribution from the inside part $B_{i n}$ will be computed in the following subsections and will depend on whether it is true, false, or degenerate vacua.

\subsection{True vacuum bubbles}

In this section we consider possible types of true vacuum bubbles. We consider the only $U_{F}>0$ so that the exterior geometry of the bubble will remain to be de Sitter (dS) space. The true vacuum bubble can be classified according to the interior geometry and the size of the bubble. The interior geometry of a true vacuum bubble can be different depending on $U_{T}=0$ (flat), $U_{T}<0(\mathrm{AdS})$, and $U_{T}>0(\mathrm{dS})$. The schematic diagrams of twelve possible types of solutions with different shapes are illustrated in Fig. 1: The figures of the first row (1-1) (1-3) in Fig. 1. A represent flat interior geometry, those of the second row (2-1) - (2-3) represent AdS interior geometry, and those of the third (3-1) - (3-3) and the fourth row (4-1) - (4-3) in Fig. 1.B represent dS interior geometry, respectively.

The cases in Fig. 1-B don't have stationary point in the action. Hence these solutions are not possible. The result is consistent with that of Ref. [27]. The authors analyzed the solution using the Israel matching condition [55].

The de Sitter background in Fig. 1 A can be naturally called "small" for the first column, "half" for the second column, and "large" for the third column. As for the true vacuum bubbles we will call the bubble to be "small", "half", and "large" if the de Sitter background is called "large", "half", and "small", respectively. With this terminology, the three diagrams (1-1), (2-1), and (3-1) in the first column correspond to the small bubble or large background, those in the second column correspond to half sized bubble or background, and the three diagrams (1-3), (2-3), and (3-3) in the third column correspond to the large bubble or small background.

The small bubble cases of (1-1), (2-1), and (3-1) were obtained in Refs. [4, 12]. The cases of (1-2), (1-3), (3-3), (4-1), and (4-3) are considered in Refs. [27, 47], with different action from 
ours, as a mechanism of reducing the cosmological constant [54]. There is no scalar field in their action and hence the junction condition was imposed on the bubble wall. In those papers, the magnitude of bubble wall's tension determines the type of solution. But we keep the tension constant in this paper.

We now compute the contribution from inside the wall $B_{i n}$. The expression depends on the size of the bubble and the geometry. For the small bubble, it is given by [12]

$$
\begin{aligned}
B_{i n} & =4 \pi^{2} \int_{0}^{\bar{\rho}} d \rho\left[\frac{\rho^{3} U_{T}-\frac{3 \rho}{\kappa}}{\left(1-\frac{\kappa}{3} \rho^{2} U_{T}\right)^{1 / 2}}-\frac{\rho^{3} U_{F}-\frac{3 \rho}{\kappa}}{\left(1-\frac{\kappa}{3} \rho^{2} U_{F}\right)^{1 / 2}}\right] \\
& =\frac{12 \pi^{2}}{\kappa^{2}}\left[\frac{\left(1-\frac{\kappa}{3} U_{T} \bar{\rho}^{2}\right)^{3 / 2}-1}{U_{T}}-\frac{\left(1-\frac{\kappa}{3} U_{F} \bar{\rho}^{2}\right)^{3 / 2}-1}{U_{F}}\right] .
\end{aligned}
$$

The formula will be used for the first and second column in Fig. 1,

For the general expression corresponding to the third column for large bubbles and the fourth row in Fig. 1, it is important to divide the integration range separately. If the dS bubble size is larger than half of its dS size as in the cases in the fourth row, the integration range of the true vacuum region in $B_{i n}$ should be divided into two parts. Likewise, if the size of dS background is large than the half of its $\mathrm{dS}$ size, the range should be also divided into two parts. For example, $B_{i n}$ in the case (4-3) is given by

$$
\begin{aligned}
B_{i n} & =4 \pi^{2} \int_{0}^{\rho_{\max }} d \rho\left[\frac{\rho^{3} U_{T}-\frac{3 \rho}{\kappa}}{\left(1-\frac{\kappa}{3} \rho^{2} U_{T}\right)^{1 / 2}}-\frac{\rho^{3} U_{F}-\frac{3 \rho}{\kappa}}{\left(1-\frac{\kappa}{3} \rho^{2} U_{F}\right)^{1 / 2}}\right] \\
& -4 \pi^{2} \int_{\rho_{\max }}^{\bar{\rho}} d \rho\left[\frac{\rho^{3} U_{T}-\frac{3 \rho}{\kappa}}{\left(1-\frac{\kappa}{3} \rho^{2} U_{T}\right)^{1 / 2}}-\frac{\rho^{3} U_{F}-\frac{3 \rho}{\kappa}}{\left(1-\frac{\kappa}{3} \rho^{2} U_{F}\right)^{1 / 2}}\right],
\end{aligned}
$$

where $\rho_{\max }(F / T)=\sqrt{3 / \kappa U_{F / T}}$ for dS geometry. This can be seen by the relation

$$
d \rho= \pm d \eta\left[1-\frac{\kappa \rho^{2} U}{3}\right]^{1 / 2}
$$

where + for $0 \leq \eta<\eta_{\max } / 2$, 0 for $\eta=\eta_{\max } / 2$, and - for $\eta_{\max } / 2<\eta \leq \eta_{\max }$. It turns out that the cases (4-1) - (4-3) in Fig. 1 don't have the stationary point in the action, allowing no solutions.

We now consider each column in more detail.

In the case of the first three rows in Fig. 1, the form of the nucleation rate and radius are mainly determined by the value $\tilde{\kappa}[20$. To obtain the bubble radius, the coefficient $B$ is demanded to have the stationary value with respect to $\bar{\rho}$ variation. There are two bubbles with the same radius formula because Euclidean de Sitter space has the topology of $S^{4}$. One is the the small bubble (the cases of (1-1), (2-1), and (3-1)) and the other is the large bubble (the cases of (1-3), (2-3), and (3-3)) depending on the relation among parameters. For the former, 
$U_{F}-U_{T}>\frac{3 \kappa S_{o}^{2}}{4}$. For the latter, $U_{F}-U_{T}<\frac{3 \kappa S_{o}^{2}}{4}$. The existence of the above two types of bubbles can also be seen numerically by giving the different value of a numerical parameter, $\tilde{\kappa}=\frac{\mu^{2}}{\lambda} \kappa$, while keeping the constant surface tension, $\tilde{S}_{o}=2 / 3$ [20]. Half bubbles are possible under the condition $U_{F}-U_{T}=\frac{3 \kappa S_{o}^{2}}{4}$. The cases in (1-1), (2-1), and (3-1) have the small numerical value of $\tilde{\kappa}$ than that of the cases in (1-3), (2-3), and (3-3). For large bubble, $\tilde{\eta}$ exceeds $\frac{\tilde{\eta}_{\max }}{2}$ while the particle remains near the true vacuum state for a long time in the inverted potential. This can be related to the fact that $\tilde{\eta}_{\max }$ becomes smaller as $\tilde{\kappa}$ becomes larger. Other point of view of varying the surface tension is in Refs. [25, 27, 47]. Although the bubbles in the first and third columns have the same formula for the radius, they have different numerical values in general.

The first column in Fig. 1. A for the cases of the cases (1-1), (2-1), and (3-1) corresponds to the small true vacuum bubble or the large false vacuum $\mathrm{dS}$ background. The small bubbles occur when $U_{F}-U_{T}>3 \kappa S_{o}^{2} / 4$. This condition corresponds to $1>\left(\frac{\bar{\rho}_{o}}{2 \lambda_{2}}\right)^{2}$. The integral range need not to be divided as in Eq. (77) and the results for these cases were already obtained by Parke in Ref. [12]

$$
\bar{\rho}^{2}=\frac{\bar{\rho}_{o}^{2}}{D}, \quad B=\frac{2 B_{o}\left[\left\{1+\left(\frac{\bar{\rho}_{o}}{2 \lambda_{1}}\right)^{2}\right\}-D^{1 / 2}\right]}{\left[\left(\frac{\bar{\rho}_{o}}{2 \lambda_{2}}\right)^{4}\left\{\left(\frac{\lambda_{2}}{\lambda_{1}}\right)^{4}-1\right\} D^{1 / 2}\right]},
$$

where $D=\left[1+2\left(\frac{\bar{\rho}_{o}}{2 \lambda_{1}}\right)^{2}+\left(\frac{\bar{\rho}_{o}}{2 \lambda_{2}}\right)^{4}\right], \lambda_{1}^{2}=\left[3 / \kappa\left(U_{F}+U_{T}\right)\right]$, and $\lambda_{2}^{2}=\left[3 / \kappa\left(U_{F}-U_{T}\right)\right] . \bar{\rho}_{o}=3 S_{o} / \epsilon$ and $B_{o}=27 \pi^{2} S_{o}^{4} / 2 \epsilon^{3}$ are the bubble radius and the nucleation rate in the absence of gravity, respectively. $\epsilon=U_{F}-U_{T}$. Here we used $\left(1-\frac{\kappa}{3} U_{F} \bar{\rho}^{2}\right)^{3 / 2}=\frac{\left[1-\left(\frac{\bar{\rho} o}{2 \lambda}\right)^{2}\right]^{3}}{D^{3 / 2}}$.

Case (2-1) and case (3-1) correspond to the small AdS and dS bubble, respectively. Case (1-1) corresponds to the small flat bubble. The radius of the bubble and the nucleation rate can be simplified after substituting $U_{T}=0$ into Eq. (10) as

$$
\bar{\rho}=\frac{\bar{\rho}_{o}}{1+\left(\bar{\rho}_{o} / 2 \lambda\right)^{2}}, \quad B=\frac{B_{o}}{\left[1+\left(\bar{\rho}_{o} / 2 \lambda\right)^{2}\right]^{2}},
$$

where $\lambda^{2}=3 / \kappa \epsilon$. The results was first obtained in Ref. [4].

The second column corresponds to the half true vacuum bubble i.e. the half false vacuum background. These cases can be obtained from Parke's results by taking the limit of the radius of the bubble $\bar{\rho}$ equal to the size of the dS background, $\bar{\rho}=\rho_{\max }(F)$. This half bubble condition gives $U_{F}-U_{T}=3 \kappa S_{o}^{2} / 4$. This condition corresponds to $1=\left(\frac{\bar{\rho}_{o}}{2 \lambda_{2}}\right)^{2}$. Under this condition, $D$ defined below Eq. (10) can be simplified as $D=\left(S_{o}^{2}+\frac{4}{3 \kappa} U_{T}\right)\left(4 / S_{o}^{2}\right)$.

If we substitute $\bar{\rho}=\rho_{\max }(F)$ into Parke's formula, the radius of the bubble and the nucleation rate can be evaluated as follows

$$
\bar{\rho}=\frac{2}{\kappa \sqrt{S_{o}^{2}+\frac{4}{3 \kappa} U_{T}}}, \quad B=\frac{2 B_{o}}{\left(\frac{\bar{\rho}_{o}}{2 \lambda_{2}}\right)^{4}\left[\left(\frac{\lambda_{2}}{\lambda_{1}}\right)^{4}-1\right] D^{1 / 2}}\left[\frac{8 U_{F}}{3 \kappa S_{o}^{2}}-D^{1 / 2}\right] .
$$

Case (2-2) and case (3-2) correspond to the half AdS and dS bubble, respectively. Case (1-2) corresponds to the half flat bubble. The radius of a bubble and the nucleation rate can 

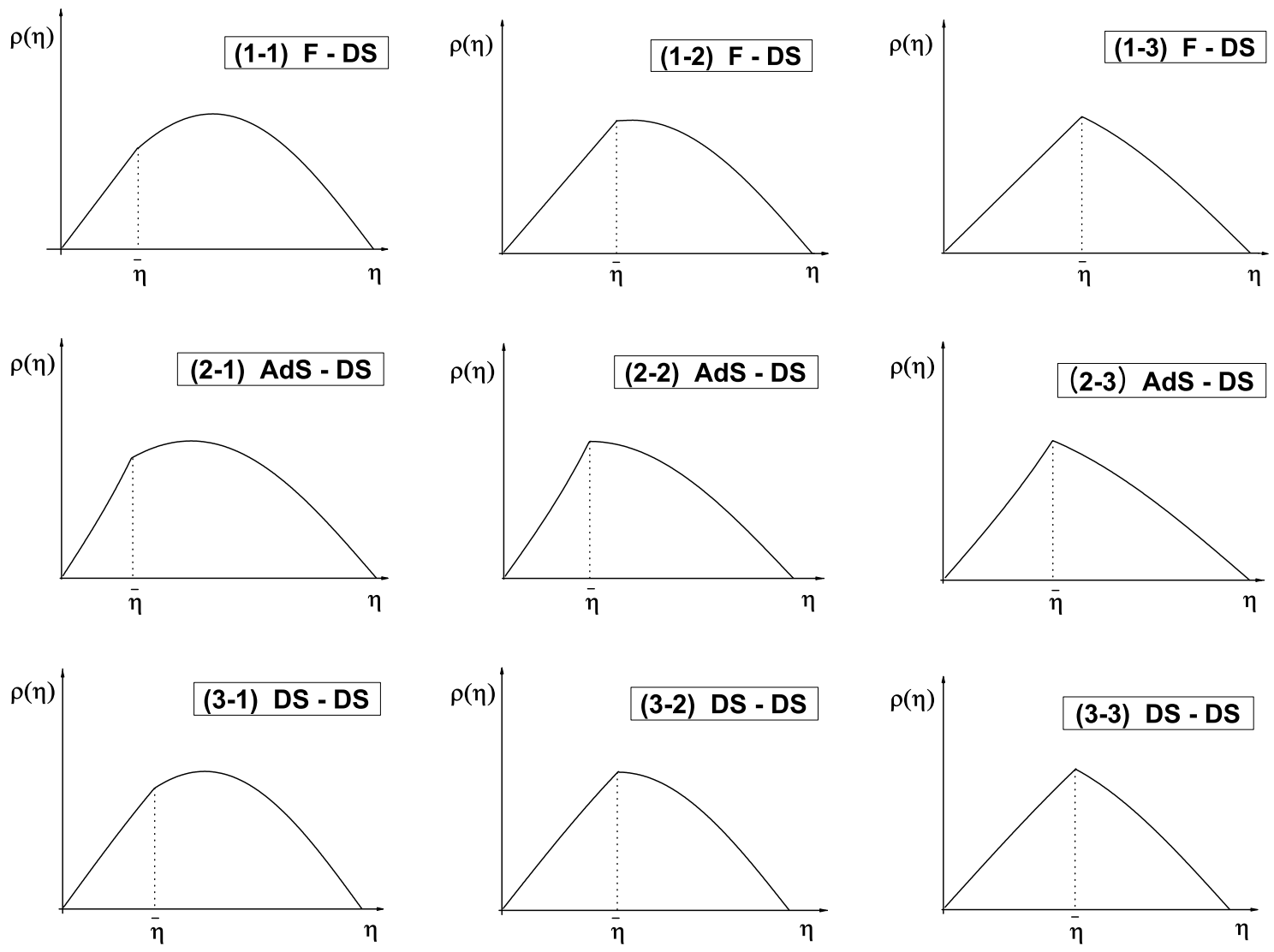

Figure 1-A
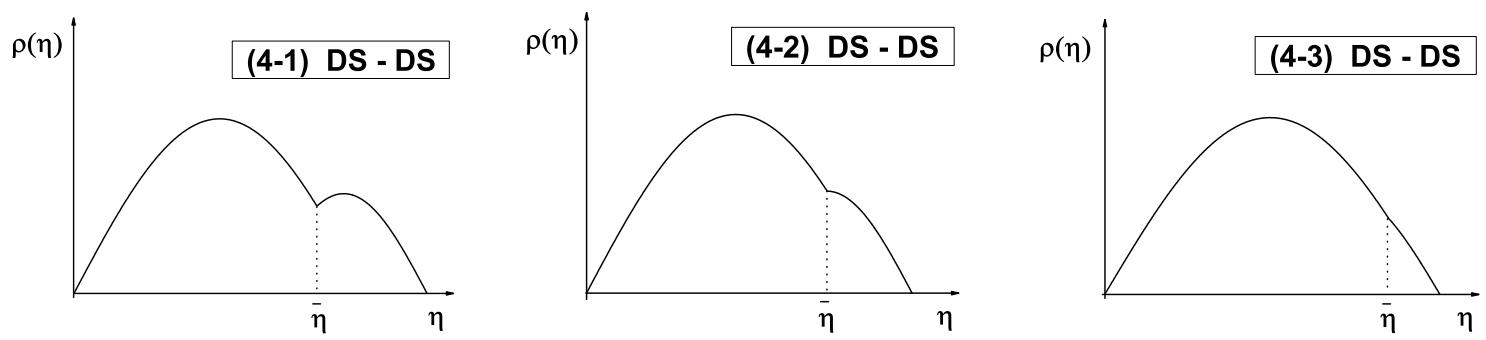

Figure 1-B

Figure 1: The schematic diagram for 12 possible types of true vacuum bubbles or matching with the thin-wall approximation. The $\bar{\eta}$ indicates the location of the wall. All the nine cases in Figure 1-A are possible solutions. The cases (4-1) - (4-3) in Figure 1-B don't have the stationary point in the action, allowing no solutions. 

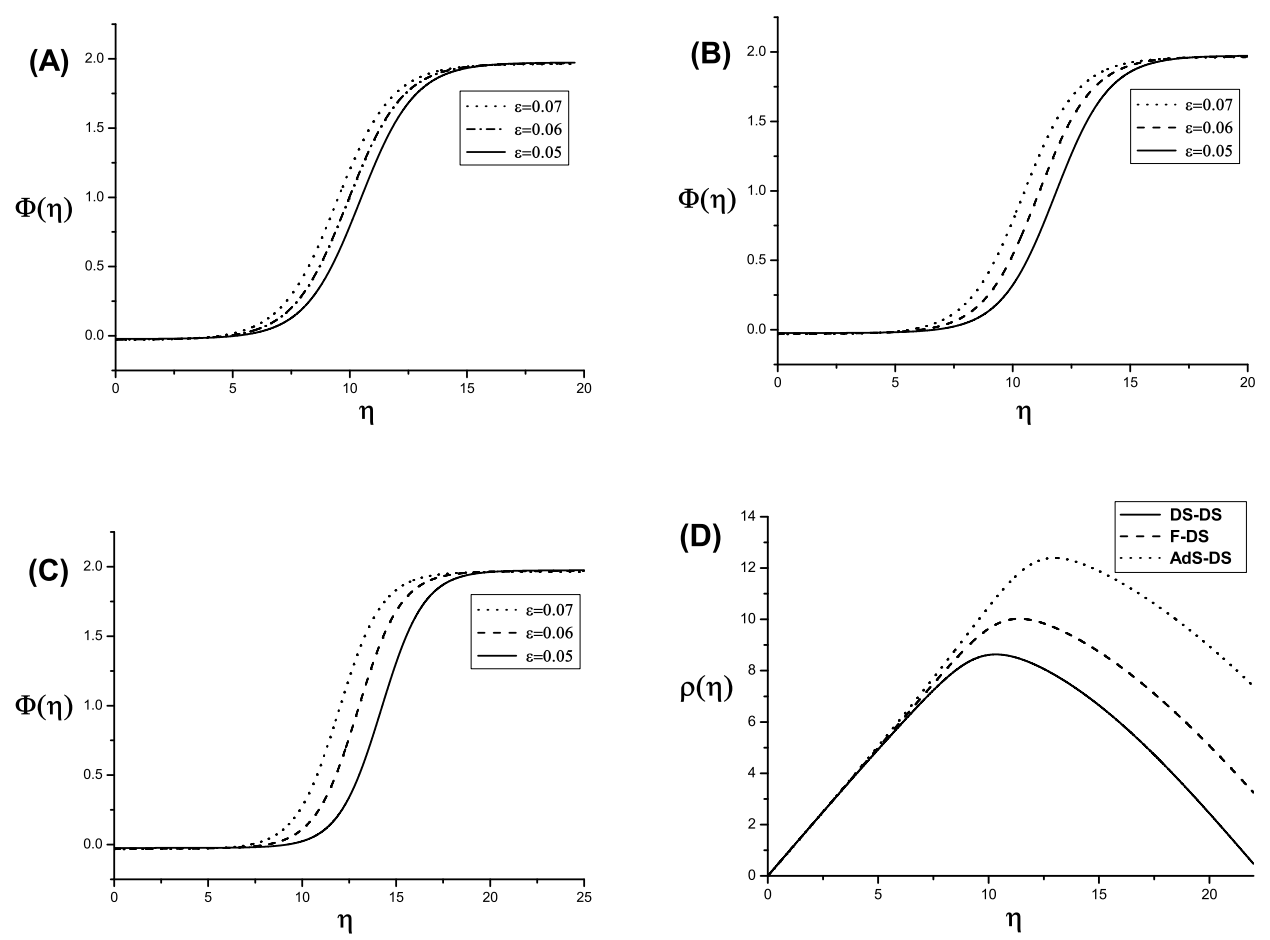

Figure 2: Numerical solutions for several types of true vacuum bubbles. We take $\tilde{\kappa} \simeq 0.3849$ for all cases. The first three figures are: (A) large flat bubble - small dS background corresponding to (1-3) in Fig. 1) (B) large AdS bubble - small dS background corresponding to (2-3); (C) large dS bubble - small dS background corresponding to (3-3). In Fig. (D), we take $\tilde{\epsilon}=0.07$. We can see from these figures that the radius of a bubble becomes larger as $\tilde{\epsilon}$ becomes smaller.

be simplified after substituting $U_{T}=0$ into Eq. (12) as

$$
\bar{\rho}=\frac{2}{S_{o} \kappa}, \quad B=\frac{8 \pi^{2}}{S_{o}^{2} \kappa^{3}} .
$$

Finally we consider the third column of Fig. 1-A corresponding to the large true vacuum bubbles i.e. the small false vacuum background. For the cases (1-3), (2-3), and (3-3) in the third column in Fig. 1, the integral range for the background should be divided into two parts, hence $B_{i n}$ is then given by

$$
\begin{aligned}
B_{i n} & =4 \pi^{2}\left[\int_{0}^{\bar{\rho}} \frac{\rho^{3} U_{T}-\frac{3 \rho}{\kappa}}{\left(1-\frac{\kappa}{3} \rho^{2} U_{T}\right)^{1 / 2}} d \rho-\int_{0}^{\rho_{\max }} \frac{\rho^{3} U_{F}-\frac{3 \rho}{\kappa}}{\left(1-\frac{\kappa}{3} \rho^{2} U_{F}\right)^{1 / 2}} d \rho+\int_{\rho_{\max }}^{\bar{\rho}} \frac{\rho^{3} U_{F}-\frac{3 \rho}{\kappa}}{\left(1-\frac{\kappa}{3} \rho^{2} U_{F}\right)^{1 / 2}} d \rho\right] \\
& =\frac{12 \pi^{2}}{\kappa^{2}}\left[\frac{\left(1-\frac{\kappa}{3} U_{T} \bar{\rho}^{2}\right)^{3 / 2}-1}{U_{T}}-\frac{\left(1-\frac{\kappa}{3} U_{F} \bar{\rho}^{2}\right)^{3 / 2}-1}{U_{F}}+2 \frac{\left(1-\frac{\kappa}{3} U_{F} \bar{\rho}^{2}\right)^{3 / 2}}{U_{F}}\right] .
\end{aligned}
$$


This is clearly different from Eq. (7).

The critical bubble size $\bar{\rho}$ can be obtained by minimizing $B=B_{i n}+B_{\text {wall }}$. The large bubbles occur when $U_{F}-U_{T}<3 \kappa S_{o}^{2} / 4$. This condition corresponds to $\left(\frac{\bar{\rho}_{o}}{2 \lambda_{2}}\right)^{2}>1$. Case (2-3) and case (3-3) correspond to the large AdS and dS bubble, respectively. It turns out that the nucleation rate and the radius are given by the same form as in Eq. (10) even though $B_{\text {in }}$ is different. Here we use $\left(1-\frac{\kappa}{3} U_{F} \bar{\rho}^{2}\right)^{3 / 2}=\frac{\left[\left(\frac{\bar{\rho}_{o}}{2 \lambda_{2}}\right)^{2}-1\right]^{3}}{D^{3 / 2}}$. Case (1-3) corresponds to the large flat bubble. The radius and the nucleation rate of a bubble have the same form as in Eq. (111).

To summarize, the results of the first column in Fig. 1] were already obtained in Ref. [12] and the results of the second column can be obtained from Eq. (10) using the half size condition $U_{F}-U_{T}=3 \kappa S_{o}^{2} / 4$. The results of the third column have the same form as in Eq. (10) even though $B_{i n}$ is different. We concluded that there is only one formula by Parke for the possible types of the true vacuum bubble regardless of the size of the bubble or different $B_{i n}$.

The true vacuum bubbles corresponding to the first three rows in Fig. 1 exist beyond the thin-wall approximation. As some examples, we present numerical solutions in Fig. 2 corresponding to (1-3), (2-3), and (3-3) in the third column of Fig. 1-A.

\section{$2.2 \quad$ False vacuum bubbles}

We now consider the nucleation of a false vacuum bubble in de Sitter background. This was first studied by Lee and Weinberg [14]. We study four cases. The three cases will correspond to the third row in Fig. 1 with $\eta \rightarrow \eta_{\max }-\eta$. The fourth case will be Hawking-Moss transition.

The first case corresponds to the reflected diagram of (3-1) in Fig. 1, The authors in Ref. [14] have obtained the nucleation of a false vacuum bubble within the true vacuum background and obtained the ratio between the decay rate of the true vacuum and that of the false vacuum. In this work, we assume the thin-wall approximation is valid. Using the approximation, we obtain the nucleation rate and the radius of a false vacuum bubble. The contribution from inside the wall is given by

$$
\begin{aligned}
B_{\text {in }} & =S^{b}(\text { in })-S^{T}(\text { in })=4 \pi^{2} \int_{0}^{\rho_{\max }} d \rho\left[\frac{\rho^{3} U_{F}-\frac{3 \rho}{\kappa}}{\left(1-\frac{\kappa}{3} \rho^{2} U_{F}\right)^{1 / 2}}-\frac{\rho^{3} U_{T}-\frac{3 \rho}{\kappa}}{\left(1-\frac{\kappa}{3} \rho^{2} U_{T}\right)^{1 / 2}}\right] \\
& -4 \pi^{2} \int_{\rho_{\max }}^{\bar{\rho}} d \rho\left[\frac{\rho^{3} U_{F}-\frac{3 \rho}{\kappa}}{\left(1-\frac{\kappa}{3} \rho^{2} U_{F}\right)^{1 / 2}}-\frac{\rho^{3} U_{T}-\frac{3 \rho}{\kappa}}{\left(1-\frac{\kappa}{3} \rho^{2} U_{T}\right)^{1 / 2}}\right] \\
& =\frac{12 \pi^{2}}{\kappa^{2}}\left[\frac{\left(1-\frac{\kappa}{3} U_{T} \bar{\rho}^{2}\right)^{3 / 2}+1}{U_{T}}-\frac{\left(1-\frac{\kappa}{3} U_{F} \bar{\rho}^{2}\right)^{3 / 2}+1}{U_{F}}\right],
\end{aligned}
$$

where we used $\rho_{\max }^{2}(F / T)=\frac{3}{\kappa U_{F / T}}$. Thus the action becomes

$$
B=\frac{12 \pi^{2}}{\kappa^{2}}\left[\frac{\left(1-\frac{\kappa}{3} U_{T} \bar{\rho}^{2}\right)^{3 / 2}+1}{U_{T}}-\frac{\left(1-\frac{\kappa}{3} U_{F} \bar{\rho}^{2}\right)^{3 / 2}+1}{U_{F}}\right]+2 \pi^{2} \overline{\rho^{3}} S_{o} .
$$


The tension of the wall is positive for the pure Einstein gravity as in the case of the true vacuum bubble. This case occurs when $U_{F}-U_{T}>3 \kappa S_{o}^{2} / 4$.

The formula for radius turns out to be the same as the case (2-1) in Fig. 1. We obtain the coefficient $B$ at this extremum

$$
B=\frac{2 B_{o}\left[\left\{1+\left(\frac{\bar{\rho}_{o}}{2 \lambda_{1}}\right)^{2}\right\}+D^{1 / 2}\right]}{\left[\left(\frac{\bar{\rho}_{o}}{2 \lambda_{2}}\right)^{4}\left\{\left(\frac{\lambda_{2}}{\lambda_{1}}\right)^{4}-1\right\} D^{1 / 2}\right]} .
$$

Here we used $\left(1-\frac{\kappa}{3} U_{F} \bar{\rho}^{2}\right)^{3 / 2}=\frac{\left[1-\left(\frac{\bar{\rho} o}{2 \lambda_{2}}\right)^{2}\right]^{3}}{D^{3 / 2}}$. As a result, the nucleation rate for the false vacuum bubble is given by changing -1 into +1 in the numerator in Eq. (10). The ratio in Ref. [14] can be obtained from Eq. (17) and Eq. (10).

The second case corresponds to the reflected diagram of (3-2) in Fig. 1. This case occurs when $U_{F}-U_{T}=3 \kappa S_{o}^{2} / 4$. The radius of the bubble has the form as in (2-2) and the nucleation rate is evaluated to be

$$
B=\frac{2 B_{o}}{\left(\frac{\bar{\rho}_{o}}{2 \lambda_{2}}\right)^{4}\left[\left(\frac{\lambda_{2}}{\lambda_{1}}\right)^{4}-1\right] D^{1 / 2}}\left[\frac{8 U_{F}}{3 \kappa S_{o}^{2}}+D^{1 / 2}\right] .
$$

This result can be obtained if one substitutes $\bar{\rho}=\rho_{\max }(F)$ into Eq. (17).

We now consider the third case corresponding to the reflected diagram of (3-3) in Fig. 1 . This case occurs when $U_{F}-U_{T}<3 \kappa S_{o}^{2} / 4$. The radius of the bubble has the form as in (2-1) and the nucleation rate has the same form as in Eq. (17). Here we use $\left(1-\frac{\kappa}{3} U_{F} \bar{\rho}^{2}\right)^{3 / 2}=\frac{\left[\left(\frac{\bar{\rho}_{o}}{2 \lambda_{2}}\right)^{2}-1\right]^{3}}{D^{3 / 2}}$.

We concluded that there is only one formula for the possible types of false vacuum bubbles regardless of the size of the bubble or different $B_{\text {in }}$ as Parke's formula.

The fourth case corresponds to Hawking-Moss transition. If we change the upper limit of integral in Eq. (16) into $\bar{\rho}=\rho_{\max }$ and eliminate the contribution of the wall we can easily get the result obtained by Hawking and Moss. The thin-wall approximation is not considered in this transition. It describes the scalar field jumping simultaneously at the top of the potential barrier. The properties of Hawking-Moss transition were studied by many authors [30, 56].

\subsection{The case of degenerate vacua}

It is possible that the tunneling occurs via the potential with degenerate vacua in de Sitter space. The numerical solution of this tunneling was obtained in Ref. [29]. This tunneling is possible due to the changing role of the second term in Eq. (3) from damping to accelerating during the transition. This case corresponds to a special transitions of the nucleation of a large true vacuum bubble and a false vacuum bubble in dS background. The solution corresponds to the particle starting near at one of two vacuum states under the influence of damping term in one half of the inverted potential, climbing the hill under the influence of accelerating term in the other half of the potential, and reaching near at the other vacuum state. It will be 
considered as the limiting case of (3-3) in Fig. 1. This solution is the instanton solution rather than the bounce solution in dS background.

Furthermore this transition is somewhat different from the formation of a domain wall. Domain walls can form in any model having a spontaneously broken discrete symmetry. The dynamical solutions with a thin domain wall was obtained by Vilenkin [57] and by Ipser and Sikivie [58]. In general, one-axis in cartesian coordinates is perpendicular to the domain wall. Thus, $z$-axis $=$ constant hypersurfaces are isometric to $(2+1)$-dimensional de Sitter space. The energy-momentum tensor is localized at the domain wall surface, $z=0$. Thus, it behaves like a delta function. The $(z, t)$-part is a $(1+1)$-dimensional Rindler space:

$$
d s^{2}=d z^{2}+(1-2 \pi G \sigma|z|)^{2}\left[-d t^{2}+e^{4 \pi G \sigma t}\left(d x^{2}+d y^{2}\right)\right],
$$

where $\sigma$ is the surface energy density. This metric has an event horizon at $z= \pm 1 / 2 \pi G \sigma$. In other coordinate system, the metric becomes

$$
d s^{2}=\left(\frac{1}{2 \pi G \sigma}\right) d \eta^{2}+\eta^{2}\left[-d t^{2}+e^{4 \pi G \sigma t}\left(d x^{2}+d y^{2}\right)\right],
$$

where Vilenkin's solution consists of two copies of the spacetime for $\eta<1 / 2 \pi G \sigma$, joined together along the wall at $\eta= \pm 1 / 2 \pi G \sigma$ [59, 60, 61]. It has the repulsive property providing a mechanism for a tunneling process, in particular a pair production of black holes.

In our case, the solution has $O(4)$ symmetry both inside and outside the wall, the location of the wall is at $\eta=$ constant. The existence of the wall doesn't affect both the inside geometry and outside one because the process doesn't change the outside geometry after the transition. Thus, the geometry depends only on the vacuum energy. The spacetimes both inside and outside of the wall are a 4-dimensional spherical Rindler type in Lorentzian signature [62]

$$
d s^{2}=d \eta^{2}+\rho^{2}(\eta)\left[-d t^{2}+\cosh ^{2} t\left(d \theta^{2}+\sin ^{2} \theta d \phi^{2}\right)\right]
$$

where $\rho(\eta)=\sqrt{\frac{3}{\Lambda}} \sin \sqrt{\frac{\Lambda}{3}} \eta$ for dS, $\rho(\eta)=\eta$ for flat, and $\rho(\eta)=\sqrt{\frac{3}{\Lambda}} \sinh \sqrt{\frac{\Lambda}{3}} \eta$ for AdS space.

If the thin-wall approximation is assumed the transition rate has the form

$$
B=\frac{24 \pi^{2}}{\kappa^{2}}\left[\frac{\left(1-\frac{\kappa}{3} U_{o} \bar{\rho}^{2}\right)^{3 / 2}}{U_{o}}\right]+2 \pi^{2} \bar{\rho}^{3} S_{o}
$$

where $U_{o}$ is minima of the potential in $\mathrm{dS}$ space, the first term is from the contribution of bulk part due to the deformed, diminished, bulk shape and the second term is from the contribution of the wall.

The nucleation rate and the critical radius of the wall are given by

$$
\bar{\rho}=\frac{2}{\kappa \sqrt{\frac{S_{o}^{2}}{4}+\frac{4}{3 \kappa} U_{o}}} \text { and } B=\frac{12 \pi^{2} S_{o}}{\kappa^{2} U_{o} \sqrt{\frac{S_{o}^{2}}{4}+\frac{4}{3 \kappa} U_{o}}} .
$$



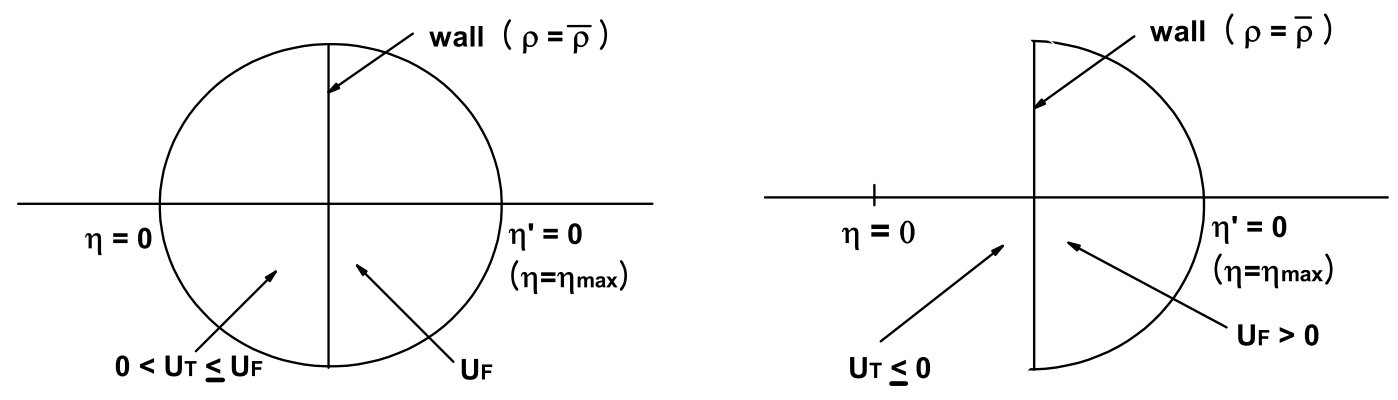

Figure 3: The schematic diagram of the bubble geometry with the wall. The left figure corresponds to the case of one half of the deformed de Sitter space and the other half of the deformed de Sitter space with same or different vacuum energy. The right figure corresponds to the case of one half of flat or anti-de Sitter space and the other half of the deformed de Sitter space.

The location of the wall is smaller than $\eta_{\max } / 2$ because the process deforms, or diminishes, the shape of $S^{4}$.

As for the consistency check, one can show that Parke's formula for the large true vacuum bubble (3-3) and Eq. (17) for the reflected diagram of (3-3) become Eq. (23) for the degenerate case if the condition $U_{F}=U_{T}=U_{o}$ is substituted into Parke's formula and Eq. (17). In other words, nucleation rates and radii of the large true vacuum bubble and false vacuum bubble are continuously connected at the degenerate case as we expected.

\section{Pair creation of black holes in the presence of a wall}

In this section we study the pair creation of black holes in the background with the wall. In Ref. [46] the authors studied pair creation of black holes in the presence of the domain wall using Euclidean junction condition with the $Z_{2}$ symmetry. The authors obtained the probability of the pair creation in the background with vanishing cosmological constant via the cut-and-paste procedure. In this work we will use vacuum bubbles obtained in Sec. 2, The Fig. 3 shows the schematic diagram of the bubble geometry with the wall. The geometry of outside the wall represented as the right half in Fig. 3 is dS, while inside geometry represented as the left half in Fig. [3] is AdS, flat, or dS depending on the cosmological constant. We define $\eta^{\prime}=\eta_{\max }-\eta$ in the right half region.

Let us consider the action

$$
S_{E}=\int_{\mathcal{M}} \sqrt{g_{E}} d^{4} x\left[-\frac{R}{2 \kappa}+\frac{1}{2} \nabla^{\alpha} \Phi \nabla_{\alpha} \Phi+U(\Phi)\right]-\oint_{\Sigma} \sqrt{h_{E}} d^{3} x \frac{K}{\kappa}+\sigma \oint_{\Sigma} \sqrt{h_{E}} d^{3} x
$$


where the last term is a Nambu-Goto-type action on the wall. Actually, the bulk park has two distinct spaces with boundaries $\Sigma_{+}$and $\Sigma_{-}$, respectively : the inside (or left) and outside (or right) of the wall. Following the work of Chamblin and Reall [63], we can obtain the Israel junction conditions as well as the Einstein equations, and scalar field equation from the above action. From the Einstein equations, the curvature scalar is obtained as $R_{E}=$ $\kappa\left[\nabla^{\alpha} \Phi \nabla_{\alpha} \Phi+4 U(\Phi)\right]$. From the junction conditions, the trace of the extrinsic curvature is obtained as $K=\frac{3}{2} \kappa \sigma$. The direction of two normal vectors is taken to be pointing outward [60, 64, 65]. In our cases two normal vectors point in the opposite direction. After rearranging the terms, the action becomes

$$
S_{E}=-\frac{\sigma}{2} \oint_{\Sigma} \sqrt{h_{E}} d^{3} x-\int_{\mathcal{M}} \sqrt{g_{E}} d^{4} x U(\Phi) .
$$

If we think over the action Eq. (24) as a plausible initial setting, we have to take into account the contribution from the relation between the boundary term and the last term. Similar type of the action also appears in the domain wall production. The action is different from that of usual bubble nucleation [4], which is slightly deviated from the case in flat spacetime [2, 3]. In the thin wall, the scalar field varies continuously between the true and the false vacuum states. Thus, the boundary term doesn't contribute to the action.

We now do the following coordinate transformations suitable for $O(3)$ invariant configurations

$$
\begin{aligned}
R & =\sqrt{3 / \Lambda} \sin (\sqrt{\Lambda / 3} \eta) \sin \chi, \quad T=\sqrt{3 / \Lambda} \tan ^{-1}(\tan (\sqrt{\Lambda / 3} \eta) \cos \chi), \text { for } \mathrm{dS} \\
R & =\eta \sin \chi, \quad T=\eta \cos \chi, \quad \text { for flat } \\
R & =\sqrt{3 / \Lambda} \sinh (\sqrt{\Lambda / 3} \eta) \sin \chi, \quad T=\sqrt{3 / \Lambda} \tanh ^{-1}(\tanh (\sqrt{\Lambda / 3} \eta) \cos \chi), \text { for AdS. }
\end{aligned}
$$

Then the metric becomes

$$
d s^{2}=\left(1 \pm \frac{\Lambda}{3} R^{2}\right) d T^{2}+\frac{d R^{2}}{\left(1 \pm \frac{\Lambda}{3} R^{2}\right)}+R^{2} d \Omega_{2}^{2},
$$

where the sign + in the parentheses denotes AdS space and - denotes dS space.

The geometry of $\mathrm{dS}$ for this work can be divided by two parts: the inside (or left) and outside (or right) of the wall. Two halves of the Euclidean dS spaces described by Eq. (27) are joined at the wall. Each one has the range $0 \leq R<\sqrt{3 / \Lambda}$ and $0 \leq T \leq \pi \sqrt{3 / \Lambda}$. We take the de Sitter period $\beta=2 \pi \sqrt{3 / \Lambda}$ and divide by 2 into the action.

For the right half, we use $\eta^{\prime}$ in Eq. (26) instead of $\eta$. The positions $\eta=0$ and $\eta^{\prime}=0$ correspond to $R=0$. Actually, the radii $\bar{\rho}$ of the inside wall and outside are same regardless of the vacuum energy in the cases we will consider. The radius $\bar{\rho}$ is related to $\bar{R}$ (the location of the wall without black holes), as we see from Eq. (26). The induced metric on the wall is given by

$$
d s_{\Sigma}^{2}=d \tau^{2}+r^{2}(\tau) d \Omega_{2}^{2}
$$


We consider several types of the configurations in Fig. 1 as the background space for black hole pair creation. In this work the general Euclidean junction condition becomes

$$
\sqrt{1-\frac{2 G M}{r} \pm \frac{\Lambda_{-}}{3} r^{2}-\dot{r}^{2}}+\sqrt{1-\frac{2 G M}{r}-\frac{\Lambda_{+}}{3} r^{2}-\dot{r}^{2}}=4 \pi G \sigma r
$$

where $S_{o}=\sigma$ and denotes the differentiation with respect to the proper time measured by the observer moving along with the wall. The signs (-) and (+) as a subscript of $\Lambda$ represent left and right spacetime, respectively. After squaring twice, we define the effective potential for Euclidean junction equation to be

$$
V_{\text {eff }}=\frac{1}{2}-\frac{1}{2}\left[\left(2 \pi G \sigma+\frac{\left(\Lambda_{+} \pm \Lambda_{-}\right)}{24 \pi G \sigma}\right)^{2} \mp \frac{\Lambda_{-}}{3}\right] r^{2}-\frac{G M}{r},
$$

with total energy is 0 . The static bubble wall solution satisfies the following conditions

$$
V_{\text {eff }}\left(r_{b}\right)=0 \text { and }\left.\frac{d V_{\text {eff }}}{d r}\right|_{r_{b}}=0 .
$$

The solution can exist at $r_{b}=3 G M$. The masses of created black holes are uniquely determined by the given cosmological constant and surface tension on the wall:

$$
M=\frac{1}{3 G \sqrt{3\left[\left(2 \pi G \sigma+\frac{\left(\Lambda_{+} \pm \Lambda_{-}\right)}{24 \pi G \sigma}\right)^{2} \mp \frac{\Lambda_{-}}{3}\right]}} .
$$

In this process the location of the wall with black holes is given by

$$
r_{b}=3 G M \quad \text { or } \quad r_{b}=\frac{1}{\sqrt{3\left[\left(2 \pi G \sigma+\frac{\left(\Lambda_{+} \pm \Lambda_{-}\right)}{24 \pi G \sigma}\right)^{2} \mp \frac{\Lambda_{-}}{3}\right]}},
$$

while the location without black holes is given by

$$
r_{w o b}=\frac{1}{\sqrt{\left[\left(2 \pi G \sigma+\frac{\left(\Lambda_{+} \pm \Lambda_{-}\right)}{24 \pi G \sigma}\right)^{2} \mp \frac{\Lambda_{-}}{3}\right]}} .
$$

What is the effect of the black holes to the location at the wall? Before the creation, the location is approximately at de Sitter horizon but inside of that. After the creation, the location is moved toward the black hole horizon.

The action for the production takes the form

$$
S_{E}=S_{E}^{(B)}-S_{E}^{(o)},
$$


where $S_{E}^{(B)}$ and $S_{E}^{(o)}$ denote the action with black holes and without black holes, respectively. Note that the nucleation rate $S_{E}^{(B)}$ of the geometry with black holes is greater than one $S_{E}^{(o)}$ without black holes.

(i) de Sitter with degenerate vacua

Firstly, we consider the case of degenerate vacua in de Sitter space. The action is given by

$$
\begin{aligned}
S_{E}^{(o)} & =-\frac{\sigma}{2} \int \sqrt{h} d^{3} x-U_{o} \int_{0}^{\tau} d \tau \int_{0}^{\pi} d \theta \int_{0}^{2 \pi} d \phi \int_{0}^{r} d r r^{2} \sin \theta \\
& =-\frac{\sigma}{2} \pi^{2} \bar{\rho}_{w o b}^{3}-\frac{\pi}{2 G} \sqrt{\frac{\Lambda}{3}} r_{w o b}^{3}
\end{aligned}
$$

where $\rho(\eta)=\sqrt{\frac{3}{\Lambda}} \sin \sqrt{\frac{\Lambda}{3}} \eta$ for $\mathrm{dS}, \rho(\eta)=\eta$ for flat, and $\rho(\eta)=\sqrt{\frac{3}{\Lambda}} \sinh \sqrt{\frac{\Lambda}{3}} \eta$ for AdS space, $\Lambda=8 \pi G U_{o}$, and the de Sitter period $\beta=2 \pi \sqrt{3 / \Lambda}$. We divided the action by 2 . Our results with vanishing cosmological constant are reduced as those in Ref. [46]. We now compare the location of a bubble wall $\bar{\rho}$ with $r_{w o b}=\frac{1}{\sqrt{\frac{\Lambda}{3}+4 \pi^{2} G^{2} \sigma^{2}}}$ in Eq. (23). These two values are the same at $\chi=\pi / 2$ and $\tau=0$ (see Eq. (26) ).

The action for the pair creation is evaluated to be

$$
\begin{aligned}
S_{E} & =S_{E}^{C}-\frac{\sigma}{2} \sqrt{1-\frac{2 G M}{r}-\frac{\Lambda}{3} r^{2}} \int_{0}^{\tau} d \tau \int_{0}^{\pi} d \theta \int_{0}^{2 \pi} d \phi r^{2} \sin \theta \\
& -U_{o} \int_{0}^{\tau} d \tau \int_{0}^{\pi} d \theta \int_{0}^{2 \pi} d \phi \int_{0}^{r} d r r^{2} \sin \theta-S_{E}^{(o)} \\
& =S_{E}^{C}-\left(4 \pi^{2} G \sigma^{2} r_{b}^{3}+\frac{\Lambda}{6 G} r_{b}^{3}\right) \frac{2 \pi r_{h} \sqrt{1-\left(9 \Lambda G^{2} M^{2}\right)^{1 / 3}}}{\left|1-\Lambda r_{h}^{2}\right|} \\
& +\frac{\sigma}{2} \pi^{2} \bar{\rho}_{w o b}^{3}+\frac{\pi}{2 G} \sqrt{\frac{\Lambda}{3}} r_{w o b}^{3},
\end{aligned}
$$

where $S_{E}^{C}$ represents the contribution due to the conical deficit angle at the black hole horizon, which has to be added in the action $S_{E}$. The contribution on the conical angle was studied in Refs. [35, 37, 41].

We adopt the periodicity of the time coordinate, by the Bousso-Hawking normalization [41] for Schwarzschild-de Sitter (SdS) space, is $\beta=\frac{4 \pi r_{h} \sqrt{1-\left(9 \Lambda G^{2} M^{2}\right)^{1 / 3}}}{\left|1-\Lambda r_{h}^{2}\right|}$ as in Ref. [66]. $r_{h}$ denotes $r_{B}$ for the black hole horizon and $r_{C}$ for the cosmological horizon, respectively. In particular, this periodicity approaches a constant value given by $\beta_{N}=\frac{2 \pi}{\sqrt{\Lambda}}$ in the Nariai Limit. The statement is related to selecting of the preferred observer having the zero acceleration. In their framework, the normalized surface gravity is obtained and the Bousso-Hawking temperature is found. There are recent studies on the thermodynamics of the SdS by Myung [67], Nariai class by Cho and Nam [68], analysis of SdS by Choudhury and Padmanabhan [69], other black holes by Park [70], and black holes in higher-order theories [71]. 
(ii) the case (1-3) in Fig. 1

This case is that the left of the wall corresponds to flat space and the right corresponds to de Sitter space. The action turns out to be

$$
\begin{aligned}
S_{E} & =S_{E}^{C}-\frac{\sigma}{4}\left(\sqrt{1-\frac{2 G M}{r}} \int_{0}^{\tau_{-}} d \tau_{-}+\sqrt{1-\frac{2 G M}{r}-\frac{\Lambda_{+}}{3} r^{2}} \int_{0}^{\tau_{+}} d \tau_{+}\right) \int_{0}^{\pi} d \theta \int_{0}^{2 \pi} d \phi r^{2} \sin \theta \\
& -S_{E}(b u l k)-S_{E}^{(o)} \\
& =S_{E}^{C}-\pi \sigma r_{b}^{2}\left[\frac{4 \pi G M}{\sqrt{3}}+\left(4 \pi G \sigma r_{b}-\frac{1}{\sqrt{3}}\right) \frac{2 \pi r_{h} \sqrt{1-\left(9 \Lambda_{+} G^{2} M^{2}\right)^{1 / 3}}}{\left|1-\Lambda_{+} r_{h}^{2}\right|}\right] \\
& -\frac{\Lambda_{+}}{12 G} r_{b}^{3}\left(\frac{2 \pi r_{h} \sqrt{1-\left(9 \Lambda_{+} G^{2} M^{2}\right)^{1 / 3}}}{\left|1-\Lambda_{+} r_{h}^{2}\right|}\right)+\frac{\sigma}{2} \pi^{2} \bar{\rho}_{w o b}^{3}+\frac{\pi}{4 G} \sqrt{\frac{\Lambda_{+}}{3}} r_{w o b}^{3}
\end{aligned}
$$

where the action for flat space is obtained as in [46].

(iii) the case (2-3) in Fig. 1

This case is that the left of the wall corresponds to anti-de Sitter space and the right corresponds to de Sitter space. The action is evaluated to be

$$
\begin{aligned}
S_{E} & =S_{E}^{C}-\frac{1}{4}\left(\sigma \sqrt{1-\frac{2 G M}{r}+\frac{\Lambda_{-}}{3} r^{2}} \int_{0}^{\tau_{-}} d \tau_{-}\right. \\
& \left.+\sigma \sqrt{1-\frac{2 G M}{r}-\frac{\Lambda_{+}}{3} r^{2}} \int_{0}^{\tau_{+}} d \tau_{+}\right) \int_{0}^{\pi} d \theta \int_{0}^{2 \pi} d \phi r^{2} \sin \theta-S_{E}(\text { bulk })-S_{E}^{(o)} \\
& =S_{E}^{C}-\pi \sigma r_{b}^{2}\left[\sqrt{\frac{1}{3}+\frac{\Lambda_{-}}{3} r_{b}^{2}} \frac{2 \pi r_{h} \sqrt{\frac{1}{3}+\frac{\Lambda_{-}}{3} r_{b}^{2}}}{1+\Lambda_{-} r_{h}^{2}}\right. \\
& \left.+\left(4 \pi G \sigma r_{b}-\sqrt{\frac{1}{3}+\frac{\Lambda_{-}}{3} r_{b}^{2}}\right) \frac{2 \pi r_{h} \sqrt{1-\left(9 \Lambda_{+} G^{2} M^{2}\right)^{1 / 3}}}{\left|1-\Lambda_{+} r_{h}^{2}\right|}\right] \\
& -\frac{r_{b}^{3}}{12 G}\left[\Lambda_{+} \frac{2 \pi r_{h} \sqrt{1-\left(9 \Lambda_{+} G^{2} M^{2}\right)^{1 / 3}}}{\left|1-\Lambda_{+} r_{h}^{2}\right|}-\Lambda_{-} \frac{4 \pi r_{h} \sqrt{\frac{1}{3}+\frac{\Lambda_{-}}{3} r_{b}^{2}}}{1+\Lambda_{-} r_{h}^{2}}\right] \\
& +\frac{\sigma}{2} \pi^{2} \bar{\rho}_{w o b}^{3}+\frac{\pi}{4 G} \sqrt{\frac{\Lambda_{+}}{3}} r_{w o b}^{3}-\frac{\Lambda_{-}}{64 G} \pi \bar{\rho}_{w o b}^{4},
\end{aligned}
$$

where $\beta=\frac{4 \pi r_{h} \sqrt{1-\frac{2 G M}{r_{b}}+\frac{\Lambda_{-}}{3} r_{b}^{2}}}{1+\Lambda_{-} r_{h}^{2}}$ as in Ref. [72].

(iv) the case (3-3) in Fig. 1

This case is that the left of the wall corresponds to de Sitter space and the right corresponds 
to de Sitter space with different vacua. The action turns out to be

$$
\begin{aligned}
& S_{E}=S_{E}^{C}-\frac{\sigma}{4}\left(\sqrt{1-\frac{2 G M}{r}-\frac{\Lambda_{-}}{3} r^{2}} \int_{0}^{\tau_{-}} d \tau_{-}\right. \\
& \left.+\sqrt{1-\frac{2 G M}{r}-\frac{\Lambda_{+}}{3} r^{2}} \int_{0}^{\tau_{+}} d \tau_{+}\right) \int_{0}^{\pi} d \theta \int_{0}^{2 \pi} d \phi r^{2} \sin \theta-S_{E}(b u l k)-S_{E}^{(o)} \\
& =S_{E}^{C}-\pi \sigma r_{b}^{2}\left[\sqrt{\frac{1}{3}-\frac{\Lambda_{-}}{3} r_{b}^{2}} \frac{2 \pi r_{h_{-}} \sqrt{1-\left(9 \Lambda_{-} G^{2} M^{2}\right)^{1 / 3}}}{\left|1-\Lambda_{-} r_{h_{-}}^{2}\right|}\right. \\
& \left.+\left(4 \pi G \sigma r_{b}-\sqrt{\frac{1}{3}-\frac{\Lambda_{-}}{3} r_{b}^{2}}\right) \frac{2 \pi r_{h_{+}} \sqrt{1-\left(9 \Lambda_{+} G^{2} M^{2}\right)^{1 / 3}}}{\left|1-\Lambda_{+} r_{h_{+}}^{2}\right|}\right] \\
& -\frac{r_{b}^{3}}{12 G}\left[\Lambda_{+} \frac{2 \pi r_{h_{+}} \sqrt{1-\left(9 \Lambda_{+} G^{2} M^{2}\right)^{1 / 3}}}{\left|1-\Lambda_{+} r_{h_{+}}^{2}\right|}+\Lambda_{-} \frac{2 \pi r_{h_{-}} \sqrt{1-\left(9 \Lambda_{-} G^{2} M^{2}\right)^{1 / 3}}}{\left|1-\Lambda_{-} r_{h_{-}}^{2}\right|}\right] \\
& +\frac{\sigma}{2} \pi^{2} \bar{\rho}_{w o b}^{3}+\frac{\pi}{4 G \sqrt{3}} r_{w o b}^{3}\left(\sqrt{\Lambda_{+}}+\sqrt{\Lambda_{-}}\right) .
\end{aligned}
$$

In this section we have obtained pair creation rate of black holes in the four cases with different background types.

\section{Summary and Discussions}

In this paper we studied the possible types of vacuum bubbles and calculated the radius and the nucleation rate. We have obtained some numerical solutions as well as analytic computation using the thin-wall approximation. We consider the only $U_{F}>0$ so that the exterior geometry of the bubble will remain to be de Sitter $(\mathrm{dS})$ space. There are nine types of true vacuum bubbles, three false vacuum bubbles, and Hawking-Moss transition in which the thin-wall approximation is not considered. We confirmed that Parke's formula is applicable to all types of true vacuum bubbles even though $B_{i n}$ is different. In addition, we obtained the single formula that applies also for all types of false vacuum bubbles. There are some conditions for classifying the bubbles. The conditions are $U_{F}-U_{T}>3 \kappa S_{o}^{2} / 4$ for small bubbles, $U_{F}-U_{T}=3 \kappa S_{o}^{2} / 4$ for half bubbles, and $U_{F}-U_{T}<3 \kappa S_{o}^{2} / 4$ for large bubbles. The large true vacuum bubble corresponds to the case (3-3) and false vacuum bubble to the reflected diagram of (3-3). The coefficient $B$ and the radius of the former is continuously connected to those of the latter.

We have discussed the special case corresponding to the tunneling of degenerate vacua in de Sitter space. This tunneling is possible due to the changing role of the second term in Eq. (3) from damping to acceleration during the transition. This solution does not have any negative mode, hence corresponds to an instanton solution rather than a bounce solution in de Sitter background. We have compared this transition with the formation of a domain wall. We have obtained the transition rate and the radius of a bubble. This solution can be applied to the 
sine-Gordan model with periodic potential. Moreover this solution can be applied to the case with a complex scalar field, which has global $U(1)$ symmetry. In the potential, the transition between different minima, by moving along the circle of minima, requires an infinite energy because of the infinite volume of the space. The kinetic term in the energy is proportional to the volume. Therefore, a change in the field in the whole space requires an infinite energy and thus the transition is impossible. However, the transition via tunneling can be possible in de Sitter space. In addition, this transition by moving along the circle will be possible because of the finite volume of de Sitter space. The process of the symmetry restoration will be also possible via the Hawking-Moss transition. It will be interesting if this transition can be embedded in the case with $S O(3)$ model as well as in the cases with gauge fields in various dimensions.

We considered the pair creation of black holes in some of these vacuum bubble backgrounds. These solutions give rise to the background for the black hole pair creation more naturally. We have obtained static bubble wall solutions of junction equation with black hole pair. The masses of created black holes are uniquely determined by given cosmological constant and the surface energy density or tension on the wall. In this work, the masses of black holes are same. Finally, we have obtained the rate of pair creation of black holes by taking the difference between the nucleation rate $S_{E}^{(B)}$ of the geometry with black holes and that $S_{E}^{(o)}$ without black holes. In this paper we considered black holes having only the same mass in pure Einstein gravity. The case with different masses can be applied. Considering other theories of gravity with the modified tension of the wall will be also interesting.

Our solutions, even if it has simple structure, can be used to understand the mechanism how the complicated spacetime structure could be created in the early universe as well as tunneling phenomena occur in the string landscape and eternal inflation.

All cases we considered have at least one de Sitter space. Thus, we now need to argue the temperature of SdS space defined by the surface gravity. Actually, the one side of the background with black holes has one horizon due to a black hole because the location of the wall for each solution is inside de Sitter horizon. In general, SdS space has two horizons. One is black hole event horizon and the other the cosmological horizon, respectively. The temperature has also two types, Hawking temperature for event horizon and Gibbons-Hawking temperature for cosmological horizon in the standard normalization, respectively. Thus, there was a difficulty determining the periodicity of the time coordinate. In other words, there is a conical singularity unless both singularity may be simultaneously removed.

Actually, the surface gravity is related to the force required at infinity to hold a test particle hovering at the horizon. In SdS, there is no asymptotic flat region and to select the preferred observer with zero acceleration is difficult. Thus, one uses the standard normalization with two different temperatures defined by two different surface gravities. In the framework of the Bousso-Hawking normalization, there is one point at which the black hole attraction and the cosmological repulsion exactly cancel out. Thus, one can define the Bousso-Hawking temperature and the periodicity of the time coordinate.

If the background becomes SdS and we can't control the singular properties an extra con- 
tribution from the boundary term due to horizons, after black hole pair creation, will appear. There is no cosmological horizon in our solution because the wall is located within the horizon. The boundary effect due to the existence of the wall appeared in Eq. (24) is already included in our solution. The contribution due to the conical angle at the black hole horizon has to be added in the action $S_{E}$.

\section{Acknowledgements}

We would like to thank Kimyeong Lee, Piljin Yi, and Hyun Seok Yang at the Korea Institute for Advanced Study for their hospitality and valuable discussions and Yun Soo Myung, Jin-Ho Cho, Chanju Kim, and Hyeong-Chan Kim for useful discussions and kind comments. We would like to thank E. J. Weinberg for helpful discussions. This work was supported by the Korea Science and Engineering Foundation (KOSEF) grant funded by the Korea government(MEST) through the Center for Quantum Spacetime(CQUeST) of Sogang University with grant number R11 - 2005 - 021. W.L. was supported by the Korea Research Foundation Grant funded by the Korean Government(MOEHRD)(KRF-2007-355-C00014). 


\section{References}

[1] J. A. Wheeler, Ann. Phys. (N.Y.) 2, 604 (1957); Geometrodynamics (Academic, New York, 1962).

[2] M. B. Voloshin, I. Yu. Kobzarev, and L. B. Okun, Yad. Fiz. 20, 1229 (1974)[Sov. J. Nucl. Phys. 20, 644 (1975)].

[3] S. Coleman, Phys. Rev. D 15, 2929 (1977); ibid. D 16, 1248(E) (1977).

[4] S. Coleman and F. De Luccia, Phys. Rev. D 21, 3305 (1980).

[5] A. D. Linde, Phys. Lett. B 100, 37 (1981); Nucl. Phys. B 216, 421 (1983); V. A. Berezin, V. A. Kuzmin, and I. I. Tkachev, Phys. Lett. B 207, 397 (1988).

[6] S. W. Hawking and I. G. Moss, Phys. Lett. B 110, 35 (1982).

[7] A. H. Guth, Phys. Rev. D 23, 347 (1981); K. Sato, Mon. Not. R. Astron. Soc. 195, 467 (1981); J. R. Gott, Nature 295, 304 (1982); A. H. Guth and E. J. Weinberg, Nucl. Phys. B 212, 321 (1983); J. R. Gott and T. S. Statler, Phys. Lett. B 136, 157 (1984); M. Bucher, A. S. Goldhaber, and N. Turok, Phys. Rev. D 52, 3314 (1995); A. D. Linde and A. Mezhlumian, Phys. Rev. D 52, 6789 (1995); L. Amendola, C. Baccigalupi, and F. Occhionero, Phys. Rev. D 54, 4760 (1996); T. Tanaka and M. Sasaki, Phys. Rev. D 59, 023506 (1998); Q.-G. Huang and S.-H. H. Tye, Int. J. Mod. Phys. A 24, 1925 (2009); A. J. Tolley and M. Wyman, J. Cosmol. Astropart. Phys. 04 (2008) 028; S. C. Park and S. Yamaguchi, J. Cosmol. Astropart. Phys. 08 (2008) 009; A. J. Tolley and M. Wyman, arXiv:0809.1100.

[8] W. A. Hiscock, Phys. Rev. D 35, 1161 (1987).

[9] V. A. Berezin, V. A. Kuzmin, and I. I. Tkachev, Rev. D 43, R3112 (1991).

[10] L. Susskind, hep-th/0302219; R. Bousso and J. Polchinski, J. High Energy Phys. 06 (2000) 006; S. Kachru, R. Kallosh, A. Linde, and S. P. Trivedi, Phys. Rev. D 68, 046005 (2003); T. Banks, M. Dine, and E. Gorbatov, J. High Energy Phys. 08 (2004) 058; B. Freivogel and L. Susskind, Phys. Rev. D 70, 126007 (2004); D. Podolsky and K. Enquvist, J. Cosmol. Astropart. Phys. 02 (2009) 007; T. Clifton, A. Linde, and N. Sivanandam, J. High Energy Phys. 02 (2007) 024; D. Podolsky, J. Majumder, and N. Jokela, J. Cosmol. Astropart. Phys. 05 (2008) 024; Y.-S. Piao, Nucl. Phys. B 803, 194 (2008).

[11] A. Vilenkin, Phys. Rev. D 27, 2848 (1983); A. D. Linde, Phys. Lett. B 175, 395 (1986); A. H. Guth, Phys. Rep. 333, 555 (2000); A. Vilenkin, gr-qc/0409055; D. I. Podolsky, arXiv:0809.2453.

[12] S. Parke, Phys. Lett. B121, 313 (1983). 
[13] V. A. Rubakov and S. M. Sibiryakov, Theor. Math. Phys. 120, 1194 (1999).

[14] K. Lee and E. J. Weinberg, Phys. Rev. D 36, 1088 (1987).

[15] A. R. Brown, S. Sarangi, B. Shlaer, and A. Weltman, Phys. Rev. Lett. 99, 161601 (2007);

A. R. Brown, arXiv:0709.3532.

[16] D. Wohns, Phys. Rev. D 78, 107702 (2008).

[17] U. Gen and M. Sasaki, Phys. Rev. D 61, 103508 (2000).

[18] S. Ansoldi, A. Aurilia, R. Balbinot, and E. Spallucci, Classical Quantum Gravity 14, 2727 (1997); S. Ansoldi and L. Sindoni, Phys. Rev. D 76, 064020 (2007).

[19] R.-G. Cai, B. Hu, and S. Koh, Phys. Lett. B 671, 181 (2009).

[20] W. Lee, B.-H. Lee, C. H. Lee, and C. Park, Phys. Rev. D 74, 123520 (2006).

[21] B.-H. Lee, C. H. Lee, W. Lee, S. Nam, and C. Park, Phys. Rev. D 77, 063502 (2008).

[22] E. I. Guendelman and N. Sakai, Phys. Rev. D 77, 125002 (2008).

[23] B.-H. Lee, W. Lee, S. Nam, and C. Park, Phys. Rev. D 75, 103506 (2007).

[24] D. Stojkovic, G. D. Starkman, and R. Matsuo, Phys. Rev. D 77, 063006 (2008).

[25] K. Marvel and D. Wesley, J. High Energy Phys. 12 (2008) 034.

[26] J.-L. Lehners and N. Turok, Phys. Rev. D 77, 023516 (2008); A. Ishida, C. Kim, Y. Kim, and O-K. Kwon, Phys. Rev. D 77, 126017 (2008).

[27] K. Marvel and N. Turok, arXiv:0712.2719.

[28] A. R. Brown and E. J. Weinberg, Phys. Rev. D 76, 064003 (2007).

[29] J. C. Hackworth and E. J. Weinberg, Phys. Rev. D 71, 044014 (2005).

[30] E. J. Weinberg, Phys. Rev. Lett. 98, 251303 (2007).

[31] S.-H. Henry Tye, D. Wohns, and Y. Zhang, arXiv:0811.3753.

[32] J. Schwinger, Phys. Rev. 82, 664 (1951).

[33] H. K. Lee and Y. Yoon, J. High Energy Phys. 03 (2007) 086; S. P. Kim and H. K. Lee, Phys. Rev. D 76, 125002 (2007); T. D. Cohen and D. A. McGady, Phys. Rev. D 78, 036008 (2008).

[34] D. J. Gross, M. J. Perry, and L. G. Yaffe, Phys. Rev. D 25, 330 (1982). 
[35] P. Ginsparg and M. J. Perry, Nucl. Phys. B 222, 245 (1988).

[36] G. W. Gibbons, in Fields and Geometry (World Scientific, Singapore, 1986).

[37] Z. C. Wu, Prog. Theor. Phys. 97, 859 (1997); ibid. 97, 873 (1997).

[38] M. Khlopov, B. A. Malomed, and Y. B. Zeldovich, Mon. Not. R. Astron. Soc. 215, 575 (1985); M. Y. Khlopov, R. V. Konoplich, S. G. Rubin, and A. S. Sakharov, Grav. Cosmol. 2, S1 (1999) arXiv:hep-ph/9912422]; H. I. Kim, Phys. Rev. D 62, 063504 (2000); M. Y. Khlopov, arXiv:0801.0116.

[39] A. Gomberoff, M. Henneaux, C. Teitelboim, and F. Wilczek, Phys. Rev. D 69, 083520 (2004).

[40] R. Bousso and S. W. Hawking, Phys. Rev. D 52, 5659 (1995); R. Bousso, ibid. D 55, 4889 (1997).

[41] R. Bousso and S. W. Hawking, Phys. Rev. D 54, 6312 (1996); ibid. D 57, 2436 (1998).

[42] F. Dowker, J. P. Gauntlett, S. B. Giddings, and G. T. Horowitz, Phys. Rev. D 50, 2662 (1994); P. Yi, Phys. Rev. D 51, 2813 (1995); J. Garriga and M. Sasaki, Phys. Rev. D 62, $043523(2000)$.

[43] R. B. Mann and S. F. Ross, Phys. Rev. D 52, 2254 (1995); R. B. Mann, Classical Quantum Gravity 14, L109, (1997); Nucl. Phys. B 516, 357, (1998); I. S. Booth and R. B. Mann, Nucl. Phys. B 539, 267 (1999).

[44] Z. C. Wu, Gen. Rel. Grav. 31, 223 (1999); Phys. Lett. B 445, 274 (1999); Mod. Phys. Lett. A 15, 1589 (2000).

[45] O. J. C. Dias and J. P. S. Lemos, Phys. Rev. D 69, 084006 (2004); V. Cardoso, O. J. C. Dias, and J. P. S. Lemos, Phys. Rev. D 70, 024002 (2004); O. J. C. Dias and J. P. S. Lemos, Phys. Rev. D 70, 124023 (2004); O. J. C. Dias, hep-th/0410294.

[46] R. R. Caldwell, H. A. Chamblin, and G. W. Gibbons, Phys. Rev. D 53, 7103 (1996).

[47] A. Maloney, E. Silverstein, and A. Strominger, hep-th/0205316.

[48] G. W. Gibbons and S. W. Hawking, Phys. Rev. D 15, 2752 (1977).

[49] C. W. Misner, K. S. Thorne, and J. A. Wheeler, Gravitation (Freeman, San Francisco, 1973).

[50] C. G. Callan and S. Coleman, Phys. Rev. D 16, 1762 (1977); E. J. Weinberg, Phys. Rev. D 47, 4614 (1993); J. Baacke and V. G. Kiselev, Phys. Rev. D 48, 5648 (1993); A. Strumia, N. Tetradis, J. High Energy Phys. 11 (1999) 023; J. Baacke and G. Lavrelashvili, Phys. Rev. D 69, 025009 (2004); G. V. Dunne and H. Min, Phys. Rev. D 72, 125004 (2005); 
H. Min, J. Phys. A: Math. Gen. 39, 6551 (2006); D. Metaxas, Phys. Rev. D 75, 065023 (2007).

[51] D. Metaxas, Phys. Rev. D 78, 063533 (2008).

[52] S. Coleman, V. Glaser, and A. Martin, Commun. Math. Phys. 58, 211 (1978).

[53] D. A. Samuel and W. A. Hiscock, Phys. Lett. B 261, 251 (1991); Phys. Rev. D44, 3052 (1991).

[54] J. D. Brown and C. Teitelboim, Nucl. Phys. B 297, 787 (1988).

[55] W. Israel, Nuovo Cimento B 44, 1, (1966); ibid. B 48, 463(E) (1967).

[56] L. G. Jensen and P. H. Steinhardt, Nucl. Phys. B 237, 176 (1984); ibid. B 317, 693 (1989);

J. Garriga and A. Vilenkin, Phys. Rev. D 57, 2230 (1998); T. Banks, arXiv:hep-th/0211160;

V. Balek and M. Demetrian, Phys. Rev. D 71, 023512 (2005).

[57] A. Vilenkin, Phys. Lett. B 133, 177 (1983).

[58] J. Ipser and P. Sikivie, Phys. Rev. D 30, 712 (1984).

[59] A. Vilenkin and E. P. S. Shellard, Cosmic Strings and other Topological Defects (Cambridge University Press, Cambridge, England, 1994).

[60] S. J. Kolitch and D. M. Eardley, Phys. Rev. D 56, 4651 (1997).

[61] R. Bousso and A. Chamblin, Phys. Rev. D 59, 084004 (1999).

[62] U. H. Gerlach, Phys. Rev. D 28, 761 (1983).

[63] H. A. Chamblin and H. S. Reall, Nucl. Phys. B 562, 133 (1999).

[64] S. K. Blau, E. I. Guendelman, and A. H. Guth, Phys. Rev. D 35, 1747 (1987).

[65] R. Bousso and A. Chamblin, Phys. Rev. D 59, 063504 (1999).

[66] A. Corichi and A. Gomberoff, Phys. Rev. D 69, 064016 (2004).

[67] Y. S. Myung, Phys. Rev. D 77, 104007 (2008).

[68] J.-H. Cho and S. Nam, J. High Energy Phys. 03 (2008) 027.

[69] T. R. Choudhury and T. Padmanabhan, Gen. Rel. Grav. 39, 1789 (2007).

[70] M.-I. Park, Phys. Lett. B 647, 472 (2007); Phys. Rev. D 77, 026011 (2008).

[71] Y. S. Myung, Y.-W. Kim, and Y.-J. Park, arXiv:0806.4452; C. Garraffo and G. Giribet, arXiv:0805.3575; R.-G. Cai and N. Ohta, Phys. Rev. D 74, 064001 (2006).

[72] J. D. Brown, J. Creighton, and R. B. Mann, Phys. Rev. D 50, 6394 (1994). 